\title{
Manufacturing of Surface Relief Structures in Moving Substrates Using Photoembossing and Pulsed- Interference Holography
}

Olivier T. Picot, Rafael Alcalá, Carlos Sánchez, Mian Dai, Nanayaa F. Hughes-Brittain, Dirk J. Broer, Ton Peijs, Cees W. M. Bastiaansen

Photoembossing is a cost-effective technique for the production of complex surface relief structures in a photopolymer film, achieved via contact-mask exposure to UVlight. Here, photoembossing is explored using interference holography with a CWlaser and a nanosecond pulsed laser. It is shown that identical surface relief structures are produced if the photopolymer film is kept in a fixed position. In the case of a moving substrate, relief structures are only obtained with the pulsed laser and the heights of the relief structures and their shape are the same as in the static experiments. This illustrates that photoembossing in combination with pulsed laser interference holography is potentially useful in the production of large area structured films using roll-to-roll processes.

\section{Introduction}

The ability to control the surface structure of thin polymer films at a micro-and nanoscale has led to many applications in the area of photonics, organic electronics, micromechanics and also tissue engineering. Different techniques are used to create surface relief structures such as mechanical embossing, injection molding or micro- and nano - imprinting. A new promising technique for the production of surface relief structures is photoembossing. The technique uses a self-developing material to generate the relief structures and therefore does not require expensive molds or wet etching steps. As a consequence photoembossing is a convenient and cost effective technique for creating surface relief structures especially in low cost, large area applications.

The photoembossing process uses a photopolymer blend consisting of a polymeric binder, a multifunctional a crylate monomer, a photoinitiator and optionally additives such as inhibitors or chain transfer agents. The mixture is processed from solution with a volatile solvent and a thin solid film is obtained, after solvent evaporation, on a rigid or flexible substrate. The relief structure is then generated in three steps: (i) the film is exposed to patterned UV light to locally activate the photoinitiator, (ii) the film is heated and (iii) the film is further cured by exposing it to a flood UV exposure and a second heating step. The relief structure develops after the patterned UV exposure by heating the film above a threshold temperature. This threshold is often a discrete thermal transition like the glass transition to increase monomer mobility and enhance polymerization. Before heating and after the first exposure, the film is in the glassy state and both monomer diffusion and chemical reactions are strongly suppressed. After heating-up, the polymerization reaction and the subsequent consumption of monomer in the exposed areas provides a driving force for the diffusion of the reactive species from the non-exposed areas to the exposed areas and the resulting mass transport generates the relief structures. Though photoembossing is convenient and cost effective compared to other 
patterning techniques, it is, in its current form, performed as a discontinuous process with static substrates. This limits production speed sand in-line processing like roll-to-roll. In order to generate a high throughput in large scale applications, an in-line photoembossing process, is envisaged and demonstrated in this communication.

The classical approach of photoembossing relies on a photomask to generate a UV-light pattern. However, the use of photomask in combination with moving substrates such as encountered in a roll-to-roll process could be a costly technological challenge. On the other hand, generating light patterns on a photoresist can also be achieved using interference holography. In the simplest case, a linearly polarized laser beam is split in two coherent laser beams with the same intensity. These are made to interfere at a certain angle $(2 \mathrm{u})$ in the sample region. As a result, a line interference pattern emerges with a period of $I / 2 \sin u, I$ being the wavelength of light. This patterning technique enables to achieve sub$\mathrm{mm}$ periodicities of the light patterns provided that the wavelength of the laser light can be selected in the UV region. More complex structures such as twodimensional lattices can be prepared using multiple beam holography or several exposure steps before heating. Here, we report the use of interference holography with a continuous wave (CW) laser and a nanosecond (ns) pulsed laser using both static and moving substrates to simulate processing conditions in a roll-to-roll process.

\section{Experimental Section}

\subsection{Photopolymer Preparation}

Poly(benzyl methacrylate) (PBMA) [Scientific Polymer Products, weight-average molecular weight $(\mathrm{Mw}) 70000 \mathrm{~g} \mathrm{~mol}$ 1], dipentaerythritol penta-/hexaacrylate (Aldrich) and Irgacure 369 photoinitiator (Ciba) were used as received. Photopolymer solutionswere prepared by dissolving the binder PBMA and the monomer in a $1: 1$ weight ratio and adding $5 \mathrm{wt} \%$ of photoinitiator, in propylene glycol monomethyl ether acetate (PGMEA). The obtained solution was spin coated on glass substrates, and the solvent was evaporated at $80 \mathrm{8C}$ for 10 min to give films with an average thickness of $10 \mathrm{~mm}$.

\subsection{Surface Relief Structuring}

Photoembossed films weremadewith twotypes of lasers: an argon CW laser with a wavelength of $11 / 4351 \mathrm{~nm}$ and a pulsed $\mathrm{Nd}$ : Yag laser coupled to second and third harmonic modules emitting $4 \mathrm{~ns}$ pulses of $355 \mathrm{~nm}$ linearly polarized light with vertical polarization (repetition rate $10 \mathrm{~Hz}$ ). In both setups, CW and pulsed laser, the beam was split into equal intensity beams which were made to interfere on the substrate. The angle between the two interfering beams was 2.58 resulting in a grating period of about $8 \mathrm{~mm}$. The exposure of the film to the UV pattern is performed in two conditions: with a static substrate and with moving the substrate (translational motion) with a speed of $0.05 \mathrm{~m}_{-} \mathrm{s}_{-} 1$. In the latter case the displacement of the substrate is done in a direction parallel to the grating vector. After exposure, the samples were heated to $808 \mathrm{C}$ during $10 \mathrm{~min}$ for development. 
The obtained structures were fixed using a flood exposure with a UV Exfo Mercury lamp (100mW_cm_2) and a 350-500nm filter, for $10 \mathrm{~min}$ at room temperature (RT) and a subsequent heating step at $808 \mathrm{C}$.

\subsection{Measurements}

The height of the relief structures was measured using a Netgra, NT-MDT atomic force microscope (AFM) in semi contact mode. Repeatability was verified by producing multiple samples, the heights were measured and scatter in the experimental data is represented by error bars in the figures. IR spectra were obtained in absorption mode using a Nicolet 8700 FT-IR spectrometer.

\section{Results and Discussion}

Surface relief structures on photopolymer films are obtained by combining photoembossing with interference holography. In a first set of experiments, an interference pattern is generated with two coherent, equal intensity, linearly polarized beams coming from a CW laser. In Figure 1, the height of the relief structures is plotted as a function of exposure energy for a static substrate ("E=0) and a moving substrate ("E=0.05m . s-1) simulating conditions in a roll-to-roll process. Relief structures are obtained with the static substrate. But moving the substrate (translational motion) during the exposure to an interference pattern from a CW laser does not produce any surface relief structures as expected. The absence of relief structures on the surface of the moving substrates is directly related to the displacement of the substrate during exposure, as light

modulation sweeps the film along the grating vector direction, leading to a homogeneous dose all over the exposed area, i.e., the whole area is exposed to the same light dose.

In a subsequent set of experiments, interference patterns are generated with a 4 ns pulsed laser. Again, a comparison is made between static and moving substrates. It is shown that the pulsed laser experiments produce

the desired relief structures in both static and dynamic substrates and this in contrast with the experiments done with a continuous laser. Moreover, Fouriertransform infrared (FT-IR) experiments indicated that degradation phenomena are hardly present in both $\mathrm{CW}$ and pulsed laser exposed samples.

In order to generate a well defined pattern of radicals and therefore create relief structures on a moving substrate, the exposure time needs to be short enough to obtain a small displacement of the substrate during exposure compared to the grating pitch. The displacement of the substrate during the exposure to a pulsed interference pattern is given by $I=E{ }^{*} t$ where $I$ is the displacement of the substrate, "E the speed of the substrate and $t$ is the pulse duration. In our

experiments, "E $0.05 \mathrm{~m}$ * $\mathrm{s}-1$ and $\mathrm{t}=4.10-9 \mathrm{~s}$ and, therefore, the substrate displacement during the pulsed exposure is only $0.20 \mathrm{~nm}$. We employed an interference pattern with a pitch of $8000 \mathrm{~nm}$ which illustrates that the displacement of the substrate during exposure is small in comparison with the pitch. The sample can be considered to be nearly static during the $4 \mathrm{~ns}$ duration of the pulse and therefore similar relief modulation is obtained in moving and static substrates. 
The above-described experimental data seem to indicate that pulsed laser interference holography is an excellent tool for generating relief structures in typical continuous film production lines. Actually, Figure 3 shows two sets of gratings recorded on a photopolymer coated flexible substrate using our pulsed laser holographic setup working at $10 \mathrm{~Hz}$ repetition rate. The exposed area was set to be square by putting a fixed window $\left(5^{\star} 5 \mathrm{~mm} 2\right)$ in the interference region. The lateral size of the window and the speed of the substrate are such that consecutive pulses cover a continuous line of stitched holographic gratings (without overlapping or unexposed areas). However, a few remarks are appropriate with respect to the experimental results presented here. In Figure 4, a typical AFM profile is shown of a surface relief structure generated in a moving substrate with a pulsed laser. A typical sinusoidal-like shape of the relief structure is observed and the height of the structure is in the order of hundreds of nanometers, which gives a rather low aspect ratio (height/pitch). In previous studies, it was already shown that this is predominantly related to the processing conditions (air environment, etc.) and/or to the chemical composition of the photopolymer. Aspect ratio can be improved dramatically and relief shape modified using optimized binders, monomers, additives and/or illumination conditions. It is anticipated that the general trends observed in these studies also apply to the systems presented here and that the shape and dimensions of the relief structures can be substantially improved. More importantly, the speed of the substrate in this study was only $0.05 \mathrm{~m}^{*} \mathrm{~s}-1$ and it seems interesting to estimate the maximum line speeds that are feasible in a rollto-roll process. Equation 2 expresses the maximum speed of the substrate "E(max) in order to fully cover a moving film with surface relief structures without overlapping of contiguous gratings. This speed is a function of the repetition rate of the laser fpulse, the diameter of the laser beam dbeam and the beam expansion factor $\mathrm{k}$. The size of the beam at the interference region where the sample is placed is denoted as dhologram, $" E(\max )=$ fpulse * dbeam * $k$ with $k=$ dhologram/dbeam

The laser used here produces light pulses with a 6_10_3m diameter beam and a repetition rate of $10 \mathrm{~Hz}$ (see Section 2), limiting the maximum speed of the substrate, without beam expansion ( $k=1)$, to $6^{*} 10-2 m^{*} s-1$. This maximum speed of the substrate can be increased in a variety of ways. For instance, the laser beamcan be expanded with the proper optical components. However, the energy at thefilm should be kept high enough to generate a high modulation surface relief grating. The laser beam used in our experiments can be expanded by a factor 3.5 leading to the optimum energy $\left(8 \mathrm{~mJ}{ }^{*} \mathrm{~cm}-2\right)$ for generating relief structures (with maximum height; see Figure 2) when the laser works at full energy. This leads to a maximum line speed of $2.1^{*} 10-1 \mathrm{~m}^{*} \mathrm{~s}-1$. Of course, a further reduction in the optimum energy for photoembossing via photopolymer optimization allows to increase dhologram and consequently the maximum line speed. The use of a more powerful or higher repetition rate laser can also lead to further increases in maximum line speeds. 


\section{Conclusion}

It was shown that we could generate surface relief structures by photoembossing in a moving photopolymer film using pulsed laser interference holography. This experimental observation indicates that relief structures can be produced in a continuous operation such as a roll-to roll process. At optimized conditions, the relief structures are nearly identical to the structures obtained with a static substrate. The system is insensitive to vibrations due to short time exposure in comparison to $\mathrm{CW}$ recording, making this interesting for industrial applications. It is also shown that the maximum speed of the substrate is rather low and limited by the laser specifications. Several routes are proposed for increasing line speeds such as photopolymer optimization, the use of higher energy and higher frequency lasers and the reduction of the laser energy required for photoembossing. 